\title{
La difusión del feminismo mexicano: el papel de las feministas en los medios de comunicación (Esperanza Brito, Elena Urrutia, Marta Lamas)
}

\author{
Nathalie Ludec ${ }^{1}$
}

Recepción: 13 de febrero de 2017 / Aprobación: 5 de junio de 2017

\section{Resumen}

Los medios de comunicación, escritos y audiovisuales, constituyen un espacio predilecto para la difusión y la recepción del feminismo. Esto es lo que propone analizar este ensayo a través del estudio y análisis de tres intelectuales feministas mexicanas: Esperanza Brito, Elena Urrutia y Marta Lamas, quienes simbolizaron la lucha por la emancipación de las mexicanas, en particular en los años de las décadas 1960 1970, en un momento en que emerge y se afirma la segunda ola del feminismo. Las tres militantes transmitieron una nueva mirada sobre la sociedad mexicana, dándoles a las mujeres un nuevo protagonismo en los contenidos redaccionales de la prensa de información general (Novedades, El Sol de México y El Universal). Hoy, Marta Lamas sigue presente en los medios, incluso audiovisuales (Televisa), y refleja el empeño constante de las feministas por difundir una perspectiva de género en el tratamiento de la información y en apoyar cualquier iniciativa que permita avanzar con la construcción de una opinión pública esclarecida y del conocimiento sobre las mujeres.

\section{Palabras clave}

Movimientos de liberación femenina; medios de comunicación de masas; prensa de información diaria; difusión de la información; México

\section{Abstract}

Print and audiovisual mass media are favorite means for the dissemination and reception of feminism. This essay proposes an analysis of the collaboration of three Mexican feminist intellectuals; Esperanza Brito, Elena Urrutia and Marta Lamas, who symbolize the struggle for Mexican women's emancipation especially in the 1960s1970s and the emergence of a second wave of feminism. The three militants offered a new view of Mexican society which gave women new protagonism in daily press coverage (Novedades, El Sol de México, El Universal). Today, Marta Lama is still present in the mass media, especially audiovisual media (Televisa), and she illustrates the constant attempts by feminists to broadcast a gender perspective in the treatment of news and to support any initative which furthers the creation of an informed public opinion on women's issues.

$1 \quad$ Francesa. Doctora en Estudios Latinoamericanos por la Universidad de París 8. Catedrática en el Departamento de Lenguas Extranjeras Aplicadas, Universidad de Rennes 2 UBL, Francia. Correo electrónico: nathalie.ludec@univ-rennes2.fr 


\section{Keywords}

Women's liberation movements; mass media; daily press; Mexico; dissemination of news

\section{Resumo}

As mídias, escritas e audiovisuais são espaços idóneos para a difusão y a recepção do feminismo. É o que se propõe analisar neste ensaio, através da colaboração de tres intelectuais feministas mexicanas: Esperanza Brito, Elena Urrutia y Marta Lamas, que simbolizaram a luta pela emancipação das mexicanas, em particular nos anos 60-70, num momento em que emerge e se afirma a segunda onda do feminismo. As três militantes transmitiram uma nova mirada sobre a sociedade mexicana, ao dar às mulheres outro protagonismo nos conteúdos redacionais da imprensa de informação geral (Novedades, El Sol de México, El Universal). Hoje Marta Lamas intervém nas mídias, como na televisão mexicana (Televisa), e traduz o esforço constante das feminsistas em difundir uma perspectiva de gênero no tratamento da informação e apoiar qualquer iniciativa que permita avanzar na construção duma opinião pública informada e do conhecimento sobre as mulheres.

\section{Palavras chave}

Movimentos de libertação feminina; mídias de comunicação; imprensa de informação diaria; difusão da informação; México

"A un pueblo no se le convence sino de aquello de que quiere convencerse" (Miguel de Unamuno, 1955, p. 555).

\section{Introducción}

Los medios de comunicación, escritos y audiovisuales, constituyen un espacio privilegiado para la transmisión de las reivindicaciones feministas fuera del círculo feminista y para la construcción de nuevos discursos sobre la relación entre los sexos, al cuestionar estereotipos y prejuicios. En el contexto mexicano, las feministas, en particular en el momento de la segunda ola, estuvieron presentes en los mayores diarios capitalinos mexicanos de la prensa de información general (Novedades, El Sol de México y El Universal). Entre ellas, destacamos tres figuras fundamentales del feminismo mexicano: Esperanza Brito (1932-2007), Elena Urrutia (1932-2015) y Marta Lamas (n. 1955).

Dieron otra mirada sobre la actualidad, privilegiando, entre los géneros periodísticos, el análisis y el comentario, construidos a partir de sucesos. La experiencia periodística y militante de intelectuales, colaboradoras feministas, sirve a un objetivo: informar y formar a la opinión pública. La tarea es tanto 
más difícil cuanto que los lectores y las lectoras están alejados de las tesis feministas.

El análisis se apoya en el examen de la prensa de información general y de programas televisivos mexicanos a través de tres ejes: la producción, la transmisión y la difusión de noticias, atravesados por la perspectiva de género. Esta triple perspectiva fundamenta algunas interrogantes: ¿Qué se publica cuando las mujeres protagonizan las noticias? ¿Cuáles son las estrategias discursivas y visuales que construyen las colaboradoras, feministas, para mejorar la visibilidad de las mujeres y sobre todo cambiar la perspectiva en el tratamiento de la información?

\section{Conceptos y contextos Las mujeres en la agenda mediática}

El Proyecto de Monitoreo Global de Medios de 2015 proporciona datos para medir la visibilidad de las mujeres en las noticas. Analiza la equidad de género en las noticias de más de 130 países de todo el mundo. Para México, llega a la conclusión siguiente:

El monitoreo de medios es una herramienta estratégica para evidenciar la exclusión de las mujeres de la agenda mediática para confirmar la concentración de medios en México y el sexismo imperante en el trato periodístico de todos los temas, lo que constituye una violación al derecho a la comunicación de la sociedad y en específico de las mujeres. ... Para muestra, ... sobre la información migratoria, ... el 91 por ciento colocó en el centro de la noticia a los hombres, dejando de lado la migración femenina ... (GMMP, 2015, p. 5).

La mujeres están presentes en las noticias, en la nota roja. En el contexto mexicano, lo ilustran los asesinatos en la fronteriza Ciudad Juárez, no esclarecidos, de más de 375 mujeres desde 1993, como lo denunció Elena Poniatowska:

El Diario y El Norte de Juárez, dos de los periódicos de Ciudad Juárez, confinaban el caso de las asesinadas y desaparecidas a la nota roja y a la publicación de fotografías muy agresivas, amarillistas, en primera plana. Ponían en la portada un tacón rojo, dando la imagen de que las mujeres eran prostitutas. En vez de sensibilizar a los lectores, los artículos reforzaban la creencia de que las mujeres son basura, llevan una "doble vida" y, por lo tanto, están expuestas a que las maten (Poniatowska, 2002, párr. 3).

Esta perspectiva, propia de la prensa amarillista, se aleja de la veracidad y cualquier objetividad, rompiendo con las exigencias de la noticia como género periodístico. Al lado de la distorsión, en la interpretación, de los sucesos, tenemos, al otro extremo, el silencio, otra modalidad para invisibilizar a las mujeres. Para muestra, el artículo de Maruja Torres, titulado "4.000 mujeres invisibles", y publicado en El País semanal, el cual se refiere a las Jornadas Feministas en Granada, de esta manera: "Ningún medio de comunicación, 
ni hablado ni escrito ni visual ni mímico ni siquiera bizco, se dignó cubrir la demostración callejera" (Torres, 2009). El silencio de los medios, aquí, se traduce como la idea de que el espacio público queda por conquistar para las mujeres. Y Maruja Torres concluye: "La próxima vez, hacedme el favor de salir desnudas y con látigos".

Estos ejemplos ilustran las conclusiones del Monitoreo que denuncian la permanencia del "sexismo de los medios, los estereotipos y prejuicios sexistas insolubles en el tiempo, el espacio y las plataformas de distribución de contenido" (GMMP, 2015).

Estas primeras aproximaciones llevan a pensar los medios, y la prensa en particular, según dos perspectivas: la prensa como "mundo común" y "espacio social", dos nociones tomadas del sociólogo de los medios Jean-Pierre Esquenazi (2002). La prensa y los medios en general reflejan valores comunes de una sociedad, su conformismo, sus prejuicios, que constituyen el "mundo común", susceptible de cambiar. Efectivamente, los medios ofrecen otro espacio, que se define como "espacio social", proporcionando perspectivas y puntos de vista nuevos sobre la realidad, una información considerada un servicio público a la sociedad. Dicho espacio social se construye en momentos precisos, históricos, marcados e influenciados por movimientos sociales, como el movimiento feminista. La perspectiva feminista, y luego de género, convierte el "mundo común" en "espacio social". Ahí entran en juego periodistas e intelectuales que cuestionan este "mundo común" y la invisibilización de las mujeres en las noticias.

\section{Darle otro protagonismo y más visibilidad: el propósito del feminismo}

Que una idea sea buena, generosa no es suficiente para imponerse. El caso del feminismo, de la segunda ola² (1960-1970), es ejemplar si consideramos su propósito: el de emancipar, liberar a las mujeres con la idea de mejorar la sociedad en su totalidad y fomentar la equidad de género. Dentro de esta perspectiva, el feminismo, valiéndose del concepto del género, interroga los roles sociales, construidos cultural e históricamente. En efecto, el concepto de género permite romper con una visión estancada de los roles sociales, del "mundo común" y anuncia una nueva era que ya formulaba Rosario Castellanos (1985) en estos versos donde abogaba por "Otro modo de ser humano y libre". La propuesta cobra una dimensión temporal y, por lo

2 "Se suele asumir que la primera ola del feminismo luchó por el sufragio y el acceso a la educación, la segunda por el acceso al espacio público y por la igualdad y la tercera por la defensa de la diversidad" (Gimeno Reinoso, 2010, p. 2). 
tanto, histórica. Las feministas participan en esta elaboración del conocimiento y la opinión pública mexicana sobre las mujeres.

\section{La emergencia del movimiento feminista en México}

¿Cómo una idea, un concepto, una nueva visión del mundo se impone? ¿Cómo adquiere fuerza? Emerge en un contexto histórico favorable, a la vez nacional e internacional. Asistimos así, en los años setenta, a la emergencia de movimientos feministas en Europa y Estados Unidos con repercusiones en México, donde el contexto político es favorable. La llegada de Luis Echeverría al poder en 1970 conlleva cambios políticos después de una profunda crisis marcada por el movimiento estudiantil de protesta de 1968, aplastado por el gobierno de Gustavo Díaz Ordaz, responsable de la represión sangrienta de la protesta del 2 de octubre, en la Plaza de Tlatelolco. El nuevo presidente es partidario de la liberalización, de las reformas sociales, de la "apertura democrática". Es en aquel entonces, en 1970, cuando aparece en México el movimiento feminista, durante ese período de supuesta apertura del sistema que favorece la expresión de grupos contestatarios y nuevas corrientes de opinión. El feminismo, importado desde Europa y Estados Unidos, entra en México por las puertas de los medios de comunicación, entre otros la prensa escrita, de información general, y gracias a la colaboración de las intelectuales en las redacciones, desde los años sesenta.

Dentro de una perspectiva internacional, el gobierno de Luis Echeverría Álvarez acoge a la primera conferencia organizada por la ONU sobre la situación de las mujeres en el mundo en la capital, del 19 de junio al 2 de julio de 1975, Año Internacional de la Mujer (AIM). Las voces a favor del evento destacan los recursos disponibles para organizar eventos, crear espacios de difusión. Al fin, la idea era aprovechar el momento para dar una visibilidad al feminismo y la prensa se posiciona como un medio predilecto para difundir nuevas ideas. De esta manera, la prensa de información general en los años sesenta y setenta, ofrece un espacio de debate, una tribuna a voces innovadoras que reflejan las nuevas preocupaciones del mundo, de la sociedad con un arraigamiento en la realidad nacional.

\section{La difusión del feminismo}

La difusión del feminismo se enmarca en un proceso de transmisión, concepto que, según Régis Debray, en su Introducción a la mediología, "tiene un horizonte histórico" y "obligatoriamente pone en juego una estrategia militante" (Debray, 2000, p. 3 y p. 11). Por eso, la difusión y la transmisión de las ideas feministas exigen estrategias, recursos humanos y técnicos que examinaremos a través de la colaboración de tres intelectuales mexicanas, Esperanza Brito, Elena Urrutia y Marta Lamas, en las mayores redacciones 
de los diarios nacionales, respectivamente en Novedades, El Sol de México y El Universal, entre 1963 y 1978.

\section{Militantes e intelectuales: Esperanza Brito, Elena Urrutia, Marta Lamas}

Esperanza Brito (1932-2007), Elena Urrutia (1932-2015) y Marta Lamas (n. 1955) son tres figuras emblemáticas de aquella época, feministas e intelectuales de la Ciudad de México, que pertenecen a las clases altas de la sociedad mexicana. Entablé relaciones con cada una de ellas, a partir de los años ochenta, y desde aquel entonces he seguido su recorrido a través de entrevistas, las cuales son historias de vida y cuyo contenido esclarece el análisis de su producción periodística. Su feminismo no resulta de una necesidad sino de una convicción personal que se construyó en el entorno familiar y, sobretodo, en el entorno académico, a través de lecturas, como lo sugieren unas de sus declaraciones.

Esperanza Brito testimonia:

Leí libros: La condición de la mujer, del Dr Havel [1961], hace un recorrido histórico de la mujer en las diferentes épocas y habla de que no tenían derechos, todas están sometidas al marido, para mí, fue una revelación, porque con este librito me di cuenta de que seguíamos igual, de que seguíamos sujetas al marido, sin derechos, bueno sí podíamos votar en esa época, ... pero no era suficiente ... Leí el de Betty Friedan [La mística de la feminidad, 1963³] que también es un detonador y yo me comparé con la mujer americana que ella describía y todas las limitaciones que tenía ... todo el rollo de Friedan. Y con este libro me acabó de pescar el feminismo (E. Brito, comunicación personal, D. F., México, 13 de julio de 2005).

Sus lecturas, en particular el libro de Betty Friedan, alimentan sus primeras reflexiones. En el primer artículo que escribe en Novedades para el Hogar, el 6 de febrero de 1963, da cuenta precisamente de ese malestar del ama de casa:

Y todos los sueños, los deseos de realizar grandes proyectos que tuvo al contraer matrimonio, se han perdido en la rutina de la vida diaria y han quedado en la superficie de la mente como otros tantos deseos frustrados. Si yo hubiera ... (Brito, 1963).

Esperanza Brito cuenta: "Mucha gente me preguntaba: ¿Por qué quieres trabajar? ¿Por qué quieres hacer cosas? Yo pasé este proceso, porque yo quería hacer cosas. Lo escribí." (E. Brito, comunicación personal, 13 de julio de 2005). Brito entra en la militancia a los 40 años y, ante la estupefacción de su familia y sus amigas, crea en 1973 el Movimiento Nacional de Mujeres, basado en un feminismo liberal y escribe en varios medios impresos.

$3 \quad$ Título original en inglés: The Feminine Mystique. 
En cuanto a Elena Urrutia, lee también, entre otras autoras, a Betty Friedan:

Se traduce al inglés y al español El segundo sexo de Simone de Beauvoir. Aparecen La condición de la mujer de Kate Mitchell [1971], Ios libros de Kate Millet [La política sexual, 1971], Germaine Greer [El eunuco femenino, 1970], Shulamith Firestone [La Dialéctica del Sexo, 1970] o Sheila Rowbotham (Urrutia, 1999, p. 427).

Para Elena Urrutia, su compromiso feminista nace de un sentimiento de injusticia en su propia familia:

Si se quiere hablar de despertar, mi detonador fue un sentimiento de injusticia que generaba necesariamente insatisfacción. ¿Por qué el trato y consideración que se me daba era diferente al que recibían mis hermanos varones?, ¿... por qué lo que en ellos se aceptaba, era inaceptable en nosotras, sus hermanas? ¿por qué nos regía un doble código moral? La acumulación de interrogantes que no tenían una clara explicación hacía que te adjudicaras la causa de tu malestar cuando todavía no podías entender lo que pronto el feminismo te aclararía: que tus problemas no eran de tu exclusividad, que no eran problemas personales sino comunes a todas las mujeres, ya que los compartías con tus congéneres; más tarde entenderías que lo personal era -es- político (E. Urrutia, comunicación personal, 12 de julio de 2003).

Elena Urrutia destaca en particular en el ámbito editorial y académico, en El Colegio de México, con el Programa Interdisciplinario de Estudios de la Mujer (PIEM), que creó e impulsó. Ambas, tanto E. Brito y E. Urrutia, de la misma generación, cuestionan su educación, su entorno: "A mí me educaron para ser una abnegada mujer mexicana”, afirma E. Brito (E. Brito, comunicación personal, 13 de julio de 2005).

En cuanto a Marta Lamas, joven feminista militante, se adhiere al feminismo en 1971 y se sitúa en la corriente marxista del feminismo. Estudiante en antropología, lee a Susan Sontag, y afirma:

El feminismo me cambió la vida. Me permitió hablar desde mí: en el sentido de que si yo, siendo una mujer privilegiada, universitaria, etc., etc., vivía ciertos tipos de opresiones y discriminaciones, poderlas asumir y desde ahí hacer una batalla que incluya a todos los demás (Cimacnoticias, 2007).

Marta Lamas sigue siendo hoy una figura emblemática del feminismo a través de sus distintas actividades, entre otras, como fundadora de la revista Debate Feminista (1992), y del Grupo de Información en Reproducción Elegida (GIRE), en 1991. Por otra parte, está muy presente en los medios, incluso de gran difusión. 


\section{La colaboración en los diarios capitalinos Novedades, El Sol de México y El Universal}

Esperanza Brito, Elena Urrutia y Marta Lamas no son periodistas, aprendieron en las redacciones. Esperanza Brito empieza su colaboración en 1963, en el suplemento Novedades para el Hogar, del diario Novedades, el mejor de aquel entonces. Escribe en la sección "Pensándolo bien" hasta 1983. Teniendo una experiencia en la prensa femenina, se dirige, pues, a las mujeres que suelen leer este tipo de suplemento. Elena Urrutia colabora en Radio Universidad a partir de 1967. En la prensa, diversifica sus colaboraciones, en las redacciones de El Nacional, El Sol y Novedades, en los suplementos culturales y, luego, en el diario progresista unomasuno, como lo declara:

También colaboré en el suplemento cultural de El Sol. Emmanuel Carballo, crítico literario muy reconocido, dirigía las páginas editoriales. El director de El Sol, Benjamín Wong, me invitó, en 1976, para que escribiera sobre las mujeres. No era por estar de moda, no, era una persona sensible, me había oído en la radio, se daba cuenta de mi interés particular por los libros y pensaba que valía la pena ocuparse del tema (E. Urrutia, comunicación personal, 12 de julio de 2005).

En cuanto a Marta Lamas, quien participó al lado de Elena Urrutia en la creación de la revista feminista FEM, en 1976, comienza a escribir en El Universal en 1977, invitada por su director, Luis Javier Solana:

En los setentas, para cuando entré yo [1977], ya el feminismo era un movimiento político conocido que hacía ruido, que tenía un trabajo serio, la revista Fem, y creo que era un poco estar en la vanguardia, entender que es un punto de vista, el feminista, que tenía que estar incluido en la discusión de los problemas del país ... este hombre Luis Javier Solana es un tipo crítico, inteligente, que entiende lo que es el feminismo, entonces yo creo que para él fue importante como decir 'yo quiero una feminista en El Universal' (M. Lamas, comunicación personal, 8 de julio de 2005).

Las tres colaboradoras insisten en la libertad que tenían para escribir, escoger los temas, proponer otro estilo: "Empecé colaborando en El Nacional, en el suplemento cultural, Revista Mexicana de Cultura, dirigido por Emmanuel Carballo, tan respetuoso, nunca me corrigió nada", afirma Elena Urrutia (E. Urrutia, comunicación personal, 12 de julio de 2005).

El trabajo redaccional incluye una relación con el destinatario a quien conocen, puesto que proviene de las clases altas y acomodadas de la sociedad mexicana, como ellas, pero que no es, a priori, un militante feminista, ni un activista de izquierda. Tanto Esperanza Brito y Elena Urrutia, en Novedades, como Marta Lamas, en El Universal, buscan captar, seducir al destinatario, con estrategias discursivas para abordar temas candentes o tabúes, relacionados con la sexualidad y el cuerpo. 
Si consideramos la morfología de los periódicos, el reparto de la información dentro de las secciones, según géneros periodísticos precisos, tiene una relación íntima con el contenido redaccional, lo que implica un contrato con el lector, el cual tiene expectativas precisas. Por ejemplo, en un suplemento Sobre el Hogar, el lector o la lectora esperan una información sobre la familia, la pareja, o consejos prácticos. Pero ocurre que se produce una ruptura de este contrato, en el momento de difundir nuevas ideas, nuevas corrientes de opinión. Es lo que pasa en el diario Novedades, bajo la pluma de Esperanza Brito y Elena Urrutia, responsables, cada una de un espacio redaccional, respectivamente el suplemento Novedades para el Hogar y el suplemento dominical, cultural, La Onda.

Esperanza Brito crea una desorientación del lector que proviene, por ejemplo, de un desfase entre el título y el contenido del mismo artículo. En el segundo artículo que escribe en Novedades para el Hogar, bajo el título "Una esposa perfecta" (30 de abril de 1963), anuncia una definición de qué es una esposa perfecta. Pero, en realidad, no existe "la esposa perfecta", afirma Esperanza Brito en una parodia de la prensa femenina. La imagen de la esposa sumisa y de la mujer adorno, siempre "arreglada, peinada y tan guapa como si fuera a una fiesta" se hace añicos bajo la pluma mordaz de Esperanza Brito. Al fin, contrariamente a lo que parece anunciar el título, "Una esposa perfecta", E. Brito cuestiona la imagen tradicional de la esposa.

Elena Urrutia, a su vez, escribe a partir de sus propios gustos por la cultura. La crítica de libros le sirve para afirmar sus compromisos con el feminismo y difundirlo: el libro se convierte en un instrumento de la militancia feminista y, a veces, se vale de técnicas de la información amarillista o de la telenovela. Para muestra, este artículo publicado bajo el título "La mujer que estuvo 60 veces en la cárcel" (Urrutia, 1974), cargado de sensacionalismo y de hechos extraordinarios, anuncia el libro autobiográfico de la militante comunista Benita Galeana, Benita en esta entradilla: "Primero niña campesina maltratada por su hermana, luego mujer juguete de los hombres, Benita Galeana vivió de fichera en un cabaret y después luchó por la reivindicación social de los humildes, al lado del Partido Comunista. Y contó su historia ...".

El contenido redaccional huye de argumentaciones teóricas estructurándose a partir de la experiencia y los gustos de cada colaboradora, como lo afirma Marta Lamas: "Yo comentaba cosas que pasaban en el país desde una perspectiva feminista y a veces, muy pocas veces, intentaba como hacer un artículo más de pensamiento, las ideas ... Casi siempre, tomaba algo que había ocurrido en la sociedad nacional” ( M. Lamas, comunicación personal, 8 de julio de 2005).

Esperanza Brito, Elena Urrutia y Marta Lamas se valieron de estos espacios periodísticos predeterminados para ofrecer nuevos temas, en sintonía con su personalidad y, sobre todo, sus compromisos feministas, que 
sobrepasan los límites ideológicos del diario y del lector. Cuestionan los roles sexuales, la sexualidad y la doble moral, entre otros temas, que se sellan alrededor del cuerpo, lugar de todas las luchas para las feministas de aquellos años. El desafío es desmontar, deconstruir, lo que Elena Urrutia, en un artículo titulado "Unas mujercitas diferentes", llama "la mística que convierte lo cultural en "natural'” (Urrutia, 1976) para mostrar que la situación de las mujeres no es "natural" sino el fruto de una "cultura". Elena Urrutia quiere significar que la sexualidad y la doble moral resultan de una cultura que no reconoce a las mujeres el derecho a disponer de su propio cuerpo. Ahora bien, defiende la idea que disponer de su cuerpo fundamenta la libertad individual y está en el centro de las reivindicaciones feministas; también, descansa sobre una anticoncepción libre y una sexualidad liberada.

Elena Urrutia afirma que la mujer como el hombre tiene deseos sexuales como cualquier ser humano, y no se reduce a un ser de servicios, para el esposo y los hijos: lo afirma en el título de un artículo, "Las mujeres también son seres humanos", donde cuenta la relación extra conyugal entre una mujer y un hombre más joven, que es el tema central del libro de Doris Lessing, El último verano de Mrs. Brown, que Elena Urrutia comenta con ironía: "La aventura (habrá que llamarlo de algún modo) de Mrs Brown resulta ser con un hombre más joven que ella. Pero existen convencionalismos amorosos y uno es que esta subespecie particular, mujer mayor-hombre joven, ha de ser desesperada y romántica" (Urrutia, 1974).

La doble moral sexual acepta con complacencia a un hombre y su joven amante, pero estigmatiza a una mujer con un joven amante. A pesar de todo, Mrs. Brown, sigue Elena Urrutia, "Se entrega con entusiasmo a su nueva vida como alguien que ha mantenido en reserva un potencial que jamás podría crecer adecuadamente, y que sólo ahora puede aflorar". Elena Urrutia cuestiona los comportamientos sexuales y, entre ellos, la legitimidad de la pulsión masculina. En un artículo titulado "¿Existe igualdad sexual?" (Urrutia, 1977), se opone a la idea de que a la mujer le interesa menos el sexo que el hombre, al afirmar de nuevo que "es cultural aquello que se tomaba por biológico. Tal es el caso de la idea de que en las mujeres la mayor intensidad del impulso sexual se da a edad más tardía que en los hombres". Sigue explicando que se trataba de proteger a las jóvenes de un riesgo de embarazo, al "enseñarles que ellas no tenían sensaciones sexuales y [al] darles todos los preceptos morales necesarios para que recordaran con seguridad la lección". En cambio, la sociedad animaba a los jóvenes a "expresar sus sentimientos sexuales".

Marta Lamas, portavoz de un feminismo radical, añade la idea de animalidad en los varones que "tienen deseos 'animales'", para caracterizar su sexualidad, la del orgullo que experimentan a través de su actividad sexual mientras que "las jovencitas no sienten nada, ni siquiera piensan en 'eso'”, en un artículo titulado "La Prostitución (2), Represión y Machismo". “Temiendo por su 'reputación'”, no ceden a las presiones de sus novios. 
Su conocimiento sexual, o sea "conservar su virginidad es su único valor" (Lamas, 1978, p. 5).

Para desmontar esta doble moral sexual, Elena Urrutia retoma, y de acuerdo con ellos, los argumentos del libro de dos autores norteamericanos, el ginecólogo William Masters y la psicóloga Virginia Johnson, precursores de la nueva sexología desarrollada en Estados Unidos alrededor de los años cincuenta-sesenta, y a quienes presenta de una manera que quiere convincente, en la entradilla de un artículo titulado "¿Existe igualdad sexual?": "Masters y Johnson vuelven a revolucionar los conceptos sexuales, y ahora se pronuncian contra la servidumbre erótica femenina, contra los sentimientos de culpa y contra la supuesta y añosa superioridad sensual masculina" (Urrutia, 1977, p. 3 у p. 16).

A lo largo de sus artículos, Esperanza Brito, Elena Urrutia y Marta Lamas, muestran que la desigualdad de las relaciones entre los sexos no es natural sino que tiene su origen en una dominación masculina inscrita en las relaciones familiares, sociales e institucionales, encaminándose hacia la perspectiva de género. Abren nuevos espacios en medios impresos para difundir los planteamientos feministas y una información sobre las mujeres que resquiebra las fronteras del "mundo común" que evocamos. Proponen un "espacio social" en las secciones que se les ofrecieron en las redacciones.

Ahora, tenemos que considerar el problema de la difusión que sigue agudo, asociado a una realidad social específica: una circulación débil de la prensa y, consecuentemente, bajas tasas de lectura. Hoy siguen vigentes las mismas preocupaciones: difundir una información veraz y objetiva sobre las mujeres y hacerlas más visibles. ¿Los nuevos medios ofrecerán más posibilidades?

\section{Medios audiovisuales actuales}

Marta Lamas sigue presente en los medios, en espacios de gran audiencia que ofrecen programas "espectáculo", de entretenimiento, para dar su opinión sobre la actualidad, la situación del país y, en particular, sobre las mujeres, favoreciendo voces alternativas, como la de las Católicas por el Derecho a Decidir (CDD). Es la invitada del programa Televisa, El Mañanero y Debatitlán, con Brozo, FOROtv, lunes a viernes, 6:30 hrs ${ }^{4}$.

Marta Lamas, "la novia" de Brozo, comenta la actualidad nacional en el noticiero matutino, en una selección de eventos, dándoles protagonismo a las mujeres. Mientras tanto, Brozo hace el idiota o el ingenuo, o la cumplimenta, o repite lo que dice Marta Lamas, pero sin opinar en realidad. Los videos se encuentran disponibles, desde 2013. El evento aquí seleccionado refleja las intenciones de Marta Lamas: "Cien Años del Primer Congreso Feminista de

4 Véase http://noticieros.televisa.com/foro-tv-el-mananero/1310/opinion-marta-lamas/ 
Yucatán; Marta Lamas habla de las Jornadas Conmemorativas de los Cien Años del Primer Congreso Feminista de Yucatán, que se realizan del 13 al 16 de enero Duración: 00:13:52 Fecha: 14. Ene. 2016". Marta Lamas presenta el programa del congreso que conmemora el primer congreso feminista en México (1916), que se celebró en Mérida (Yucatán). Concluye afirmando que 100 años después de este congreso, durante el cual se planteó el respeto por los derechos sexuales y reproductivos, siguen la misma lucha, la misma reivindicación, que sólo se hizo realidad en la Ciudad de México, con la ley de La interrupción legal del embarazo (ILE), del 24 de abril de 2007.

La última parte del programa se dedica a un capítulo de la serie animada Catolicadas, que Marta Lamas promovió. Es una serie animada realizada por las Católicas por el Derecho a Decidir (CDD), que se definen de esta manera, en su página web:

Católicas por el Derecho a Decidir/ México es una organización sin fines de lucro, integrada por personas católicas, que defiende los derechos humanos de mujeres y jóvenes, en especial sus derechos sexuales y reproductivos, incluyendo el acceso al aborto seguro y legal, desde una perspectiva ética, católica y feminista, en el marco del Estado laico ${ }^{5}$.

Se encargó también de la promoción de este programa Elena Poniatowska:

El programa Catolicadas es un invento de María Consuelo Mejía quien dirige desde hace 20 años a Católicas por el Derecho a Decidir. María Consuelo es el azote de obispos y sacerdotes ... . Por lo pronto, los religiosos progresistas de México la apoyan en todas sus iniciativas. Los obispos convencionales le temen, porque María Consuelo critica y da a conocer la falta de congruencia de las enseñanzas y actitudes de nuestros obispos con la tradición católica y los documentos oficiales de la Iglesia, y señala sus fallas en los problemas de derechos humanos y, lo que es más importante para nosotras las mujeres, derechos sexuales y reproductivos (Poniatowska, 2014).

La serie Catolicadas nació el 8 de marzo de 2012 en un contexto particular, marcado por el viaje del papa Benedicto XVI y las elecciones presidenciales. La serie lleva 17 temporadas:

En Catolicadas, Sor Juana y el padre Beto transmiten los mensajes. Son los dos personajes de una parroquia imaginaria. El padre Beto, sacerdote conservador, terco y malhumoriento, y Sor Juana, una monja carirredonda, progresista y atrevida; el padre Beto encarna la postura de los obispos y Sor Juana transmite los mensajes de Católicas por el Derecho a Decidir y de la teología contemporánea. Muchas de las historias parten de sucesos reales que la gente le hace llegar a la agrupación para que se conviertan en un capítulo de Catolicadas, contado en un lenguaje sencillo, con sentido común y, sobre todo, buen humor (Poniatowska, 2014). 
Por otra parte, las Católicas por el Derecho a Decidir lanzan campañas, elaboran encuestas y se aprovechan de la internet para una difusión amplia retomando los temas de los años setenta, cuando producían folletos, sobre el tema de los derechos sexuales y reproductivos. El folleto titulado Y María fue consultada para ser madre de Dios, se elaboró en 1988 y hoy alcanza una amplia difusión en el formato electrónico actual6.

Paralelamente las Católicas por el Derecho a Decidir colocaron un cartel en las calles de México, que rezaba también: Y María fue consultada para ser madre de Dios. Comenta Elena Poniatowska, compartiendo la perspectiva: "Si a la propia virgen María se le preguntó si quería ser madre de Jesús, ¿por qué a millones de católicas se les niega ese derecho?" (Poniatowska, 2014).

\section{Recepción: ajustes y desajustes}

La recepción de los mensajes periodísticos, televisivos y electrónicos, es difícil de medir. ¿Habrá una adecuación entre lo que esperan los destinatarios y el contenido informativo difundido en la prensa, en televisión y en Interent? La pregunta refleja la dificultad de delimitar y definir lo que es la opinión pública: no existe, según P. Bourdieu (1973). Intentemos unas incursiones en medidas posibles de la opinión pública que nos vuelve al "mundo común", sin retomar aquí los debates sin fin sobre la naturaleza de la opinión pública (Laborie, 2010, p. 803).

Las colaboradoras esbozan una tipología imaginada de las reacciones ante las reivindicaciones de las mujeres de parte del lector de la prensa de información general de los años setenta. De antemano, expresan un sentimiento común de un grupo social que comparte una opinión (Laborie, 2010, p. 807).

Las reivindicaciones feministas suscitan incomprensión, sorpresa, irritación. Las declaraciones siguientes, sacadas del libro de caricaturas, Mujercitas, de la española Nuria Pompeia, presentado por E. Urrutia, en un artículo titulado "Unas mujercitas diferentes", reflejan cierto enojo:

'¡Pero si ahora las mujeres están mejor que nunca!'... (se lee en el globito que escapa de su cabeza en la primera página); 'No sé qué más quieren'; 'En mis tiempos sí que lo pasaban mal y nadie se quejaba...'; 'y antes mucho peor'...'No hay más que leer la Biblia'.

Para Elena Urrutia, se oye "la voz inflexible y recalcitrante de la vox populi", que pretende que son los hombres las víctimas en un sistema dominado por los valores feministas (Urrutia, 1976).

6 Véase http://catolicasmexico.org/ns/?cause=maria-fue-consultada-para-ser-madre-de-dios 
Otros, "Los sorprendidos no comprenden, tal vez porque no quieren, la razón de las protestas femeninas ..., se niegan a ver la realidad presente a su alrededor, qué es lo que la mujer exige, cuál es la emancipación que busca ...", afirma Brito en un artículo titulado "Revolución revivida" (Brito, 1970). Esperanza Brito sigue apuntando a "Los desconsolados, o francamente irritados, [que] no se preguntan qué puede desear la mujer; para ellos la mujer no tiene derecho a pedir o exigir nada, sino tan sólo a agradecer lo que buenamente se le quiera dar" (Brito, 1970).

Conscientes de estos obstáculos, desarrollan estrategias discursivas para explicar, argumentar, legitimar las reivindaciones de las mujeres, mostrando que todos los avances sirven para todos. Por lo menos, sembraron dudas, interrogaciones que pudieran favorecer una evolución de mentalidades, por ínfima que pudiese parecer. Evidentemente es difícil medir la influencia de la prensa, y de los medios en general, sobre la opinión pública, pero podemos observar la prensa y analizar los efectos que pretende alcanzar, sin pretender claro, dar cuenta de los efectos que pudiera producir (Charaudeau, 2005, p. 220). Con todo, hoy, nuevos recursos son disponibles para medir la recepción de los contenidos, gracias a las TIC.

Al consultar el número de visitas a la serie Catolicadas, podemos afirmar, con Elena Poniatowska (2014), que "Sor Juana es hoy la monjita más popular de México": "Desde su lanzamiento hasta la fecha, los episodios se han reproducido (3 millones 772 mil 320 veces en YouTube y 170 mil 973 personas indicaron me gusta en la página de Facebook de Católicas por el Derecho a Decidir que divulga la serie. Un éxito rotundo". Por otra parte, Televisa es el mayor canal de televisión en México. En relación con los comentarios de los internautas, para muestra "Catolicadas-Capítulo 2: Gozo y esperanza (2: 4)" recibió 677 comentarios. Añadamos que la lectura de historietas en México es un modo de lectura muy presente entre la población mexicana, sobre todo joven y las historietas tienen una difusión mucho mayor a la de la prensa informativa. ¿El éxito de la serie, medido a partir del número de visitas y los comentarios, reflejará el alejamiento de los católicos con los preceptos de la Iglesia?

El éxito de la serie animada sugiere el desajuste entre las prácticas de los católicos y los preceptos de la Iglesia católica, como lo muestran los resultados de la encuesta nacional de 2014 realizada por las CDD acerca de la "Población residente en las viviendas del país de 18 años y más, que se auto adscribe como católica". La metodología7, así como los resultados ${ }^{8}$, se

7 Católicas Por el Derecho a Decidir, Encuesta nacional de Opinión Católica 2014. Metodología, en http://encuesta.catolicasmexico.org/es/?page_id=645)

8 Católicas Por el Derecho a Decidir, Encuesta nacional de Opinión Católica 2014. Resultados, en http://encuesta.catolicasmexico.org/es/?page_id=2152) 
pueden consultar en línea, repartidos en capítulos: "Identidades católicasEstado laico-Derechos sexuales y reproductivos de adolescentes-Derechos reproductivos y Estado-Derechos de las Mujeres y Aborto-Derechos de homosexuales y lesbianas-Expectativas de cambio hacia la Iglesia".

Los resultados evidencian un malestar en la relación entre la Iglesia católica y los fieles. Para muestra, la "Opinión sobre el aborto" ${ }^{\text {p pone de }}$ relieve el irrespeto del precepto católico, que condena rotundamente el aborto en cualquier circunstancia: un $80 \%$ de los sondeados está de acuerdo con que una mujer pueda hacerse un aborto cuando la vida de la mujer está en peligro. Los datos recopilados reflejan la evolución de una sociedad, de un grupo en particular que vive en una realidad compleja, muy alejada de la percepción dogmática de la Iglesia.

La encuesta revela la tendencia del colectivo católico, que asume su disidencia. Por lo tanto el discurso de las CDD se ajusta a esta tendencia. El reto, para las CDD, no es sólo cambiar el rol de las mujeres en la jerarquía católica sino defender una teología feminista, que rechaza ese ideal femenino sacralizado que nada tiene que ver con la mujer de carne y hueso. Las CDD participan de una hermenéutica ${ }^{10}$ desarrollada por la teología feminista, que es "una teología hecha desde el otro lado del poder, una interpretación del Evangelio y las Santas Escrituras hecha con miradas de mujeres, y de mujeres comprometidas con la justicia", según María Consuelo Mejía, la directora de CDDM (Calle Rivaz y Lanza, 2004).

\section{Conclusión}

Los contenidos discursivos publicados en la prensa, en los medios audiovisuales así como en los nuevos medios, rompen unos tabúes (la sexualidad, la doble moral), y contribuyen a reflexionar sobre la realidad cotidiana con otra mirada, feminista, con un lenguaje que huye de la jerga feminista o militante. Tal perspectiva cuestiona las costumbres, las tradiciones, deconstruye los prejuicios y estereotipos: es la de la tres colaboradoras, Esperanza Briton Elena Urrutia y Marta Lamas, precursoras de la perspectiva de género y luego la de otros espacios, en televisión y en Internet. Las colaboradoras interpelan así a los lectores y a las lectoras, no frontalmente sino con estrategias discursivas. En televisión, la estrategia se basa en el espectáculo y el divertimiento. Los nuevos medios hacen posible, con un feed back casi inmediato, la intervención del destinatario, lo que proporciona un estado de la opinión, del pensamiento colectivo tan difícil de medir. Los contenidos periodísticos, televisuales o electrónicos no intentan convencer, sino favorecer o despertar

9 Católicas Por el Derecho a Decidir, Encuesta nacional de Opinión Católica 2014. Resultados; "Derechos d elas Mujeres y aborto", en http://encuesta.catolicasmexico.org/es/?page_id=2343

10 Interpretación sistemática de los textos y símbolos de la Biblia. Véase Aquino y Támez, 1998. 
algún escepticismo ante las experiencias diarias, personales y colectivas, del "mundo común". Hoy, la prensa escrita ya no es un canal predilecto para escribir con una perspectiva de género o sobre el feminismo. Los medios visuales y electrónicos prevalecen para alcanzar a un público más amplio, más joven, para construir un "espacio social" público (Habermas, 1978), que combate el lenguaje sexista y promueve la equidad de género. Podemos sostener que, al combatir el conservadurismo y la ignorancia, las feministas mexicanas han logrado crear y desarrollar vínculos con la sociedad, dando un paso más en la construcción de una opinión pública esclarecida.

\section{Referencias}

Aquino, Ma. Pilar y Támez, Elsa. (1998). Teología Feminista Latinoamericana. Universidad Politécnica Salesiana (UPS), Ecuador: Abya-Yala.

Barbieri, Teresita. (1986). Nuevos sujetos sociales: la presencia política de las mujeres en América Latina. Nueva Antropología, Revista de Ciencias Sociales, "Estudios sobre la mujer: problemas teóricos", 8(30), México, D. F.

Bourdieu, Pierre. (1973). L'opinion publique n'existe pas. Les temps modernes, 318, Paris.

Brito, Esperanza. (6 de febrero de1963). La Madre, Centro del Hogar. Novedades para el Hogar. México, D. F.

Brito, Esperanza. (30 de abril de 1963). Una esposa perfecta. Novedades para el Hogar. México, D. F.

Brito, Esperanza. (11 de setiembre de 1970). Revolución revivida. Novedades para el Hogar. México, D. F.

Calle Rivaz, Ivana y Lanza, Teresa. (8 de setiembre de 2004). Feminismo y religión católica: ver a Dios con ojos de mujer. La Cuchara. Sección Modemmujer. Méxic, D. F.

Castellanos, Rosario. (1985). Meditación en el umbral. Antología poética. México: Fondo de Cultura Económica.

Católicas por el Derecho a decidir (CDD). Catolicadas. Recuperado de http:// catolicasmexico.org/ns/?page_id=4464

Católicas por el Derecho a decidir (CDD). Capítulo 1: Una mejor iglesia. Recuperado de https://www.youtube.com/watch?v=HcXOlv2zLm4

Católicas por el Derecho a decidir (CDD). Capítulo 2: "Gozo y esperanza". Recuperado de https://www.youtube.com/watch?v=ea_GhZ-DeiE,)

Católicas por el Derecho a decidir (CDD). Encuesta nacional de opinión católica 2014. Recuperado de http://encuesta.catolicasmexico.org/es/ 
Católicas por el Derecho a decidir (CDD). Libertad de conciencia, Y María fue consultada para ser madre de Dios. Recuperado de http://catolicasmexico.org/ns/?cause=maria-fue-consultada-para-ser-madre-de-dios

Charaudeau, Patrick y Maingueneau, Dominique. (2005). Les médias et l'information, l'impossible transparence du discours. Bruxelles Bry-surMarne (Val-de-Marne): De Boeck: INA.

Cimacnoticias. (25 de abril de 2007). Un logro, despenalización del aborto: Marta Lamas a Página/12. México, D. F. Recuperado de http://www. cimacnoticias.com.mx/node/57062

Debray, Régis. (2000). Introduction à la médiologie. Paris: PUF.

Esquenazi, Jean Pierre. ( 2002). L'Écriture de l'actualité. Pour une sociologie du discours médiatique. Grenoble: Presses universitaires de Grenoble.

FOROtv El Mañanero. (s.f.). Televisa, El Mañanero y Debatitlán, con Brozo, FOROtv, Lunes a Viernes, 6:30 hrs. Recuperado de http://noticieros. televisa.com/foro-tv-el-mananero/

Galán, Lola. (21 de febrero de 2016). Radiografía de 1.444 quejas. El País. Recuperado de http://elpais.com/elpais/2016/02/20/opinion/1455966648_206949.html

Gallego-Diaz, Soledad. (27 de diciembre de 2009). La mitad del cielo. El País. Recuperado de http://www.elpais.com/articulo/opinion/mitad/cielo/elpepusocdgm/20091101elpdmgpan_6/Tes

Gimeno Reinoso, Beatriz. (12 de enero de 2010). ¿Qué feminismo? Ciudad de Mujeres. Recuperado de http://www.ciudaddemujeres.com/articulos/Que-es-feminismo

GMMP [Global Media Motitoring Project]. (2015). Proyecto de Monitoreo Global de Medios de 2015. Recuperado de http://whomakesthenews. org/gmmp/gmmp-reports/gmmp-2015-reports

GMMP [Global Media Motitoring Project]. (2015). México Proyecto de Monitoreo Global de Medios. Informe Nacional. Recuperado de http://cdn.agilitycms. com/who-makes-the-news/Imported/reports_2015/national/Mexico.pdf

Habermas, Jürgen. (1978). L’Espace public. Paris: Payot.

Havel, Jean-Eugène. (1961). La condition de la femme. Paris: A. Colin.

Laborie, P. (2010). Opinion publique. En Christian Delacroix, François Dosse, Patrick Garcia \& Nicolas Offenstadt (Dirs.), Historiographies, /l Concepts et débats. Paris: Gallimard. 
Lamas, Marta. (14 de noviembre de 1978). La Prostitución (2), Represión y Machismo". El Universal. México, D. F.

Lamas, Marta. (1986). La antropología feminista y la categoría de "género". Nueva Antropología, Revista de Ciencias Sociales, "Estudios sobre la mujer: problemas teóricos", 8(30). Recuperado de http://redalyc.uaemex.mx/redalyc/src/inicio/ArtPdfRed.jsp?iCve=15903009

Montero, Rosa. (17 de enero de 2016). Gracias. El País Semanal. Recuperado de http://elpais.com/elpais/2016/01/13/eps/1452700312_399393.html

Poniatowska, Elena. (10 de agosto de 2014). Veinte años de católicas por el Derecho a Decidir. La Jornada. Recuperado de http://www.jornada. unam.mx/2014/08/10/opinion/a05a1cul

Scott, Joan W. (1990). El género: una categoría útil para el análisis histórico. En James S. Amelang y Mary Nash (Coords.), Historia y género: las mujeres en la Europa Moderna y Contemporánea (pp. 23-58). Valencia. Recuperado de http://www.amdh.org.mx/mujeres/menu_superior/Doc_basicos/5_biblioteca_virtual/2_genero/7.pdf

Televisa, El Mañanero y Debatitlán, con Brozo. (14 de enero, 2016). Cien Años del Primer Congreso Feminista de Yucatán. Marta Lamas habla de las Jornadas Conmemorativas de los Cien Años del Primer Congreso Feminista de Yucatán, que se realizan del 13 al 16 de enero. Recuperado de http://noticieros.televisa.com/foro-tv-el-mananero/1601/cien-anosprimer-congreso-feminista-yucatan/

Torres, María. (27 de diciembre, 2009). 4.000 mujeres invisibles. El País semanal. Recuperado de http://www.elpais.com/articulo/portada/4000/ mujeres/invisibles/elpepusoceps/20091227elpepspor_1/Tes

Urrutia, Elena. (29 de setiembre de 1974). La mujer que estuvo 60 veces en la cárcel. Novedades. Suplemento La Onda, sección "Libros". México, D. F.

Urrutia, Elena. (22 de diciembre de 1974). Las mujeres también son seres humanos. Novedades. Suplemento La Onda, sección "Libros". México, D. F.

Urrutia, Elena. (19 de diciembre de 1976). Unas mujercitas diferentes, Novedades. Suplemento La Onda, sección "Libros". México, D. F.

Urrutia, Elena. (21 de agosto de 1977). ¿Existe igualdad sexual? Novedades, Suplemento La Onda, sección «Libros». México, D. F.

Urrutia, Elena. (1999). Una búsqueda de identidad y sus derroteros. En Roland Forgues (Coord.). Mujer, Creación y problemas de identidad en América Latina. Mérida, Universidad de los Andes: Consejo de Publicaciones. 